\title{
Experimental Study on Pitching Motion Mode of a SWATH-USV
}

\author{
Zhang Wenxuan, Yu Yongqiang, Gao Shasha, Yang Songlin, Cheng Zhanyuan \\ School of Naval Architecture \& Ocean Engineering, Jiangsu University of Science and Technology, Zhenjiang, China
}

Email address:

359194612@qq.com (Zhang Wenxuan)

\section{To cite this article:}

Zhang Wenxuan, Yu Yongqiang, Gao Shasha, Yang Songlin, Cheng Zhanyuan. Experimental Study on Pitching Motion Mode of a SWATH-USV. International Journal of Transportation Engineering and Technology. Vol. 4, No. 2, 2018, pp. 43-49.

doi: $10.11648 /$ j.ijtet.20180402.14

Received: October 29, 2018; Accepted: December 10, 2018; Published: December 27, 2018

\begin{abstract}
The importance of the research about ship motion model has become more prominent with the improvement of economic level. Mastering ship motion model is a necessary basis for studying ship's divisibility. The hydrodynamic parameters obtained by parameter identification methods are more precise, which is dissimilar from the parameters obtained by classical methods. In this paper, a SWATH-USV was taken as the research object to accomplish a series of pitch decay tests on the condition that the SWATH-USV was in different drafts and tilts angles in the hydrostatic state. And a mathematical model by the method of system identification based on genetic algorithms has been established, adopting VB language to program a systematic identification software. With this, the author obtained the pitch motion differential equations, and the changing regularities of hydrodynamic coefficients with draft and initial tilt angle of the model in different drafts and initial tilt angles and their kinematic equation. Using this equation to predict, the results are proved to be well matched with the experimental results. And the feasibility of the identification method is also confirmed. The analysis results and methods can provide essential technical support for the in-depth analysis of the wave resistance and the design of the boat.
\end{abstract}

Keywords: SWATH-USV, Pitching Motion Mode, System Identification, Genetic Algorithms

\section{Introduction}

It's indicated that a ship will motivate a movement which has six degrees of freedom in waves, when it is sailing on the ocean. On this account, ships will produce large pendulation. Among that, pitching is the major factor that influences navigation performance in bad weather, and there are few effective methods of anti-pitching at present.

It's indispensable that USV must possess good seakeeping qualities, for it needs to overcome the unknown external interference to perform the task independently, and the functional modules will be changed frequently. The pitch motion often accompanies multifarious accidents, such as increasing resistance, ship stalling, slamming on the bottom of the ship's side, wave on the deck, and propeller emergence, which not only bring tremendous difficulties to the ship's control, but also lead to irretrievable disasters and cannot guarantee the navigation safety.

Currently, the research on ship rolling has reached an extremely mature stage. However, as to studies on the pitch motion, it's still in its infancy due to its complex hydrodynamic characteristics. The emphasis of the ship swaying research subjects is to lucubrate for acquiring means of safeguarding ship maneuverability [1]. At present, some researchers use time series method to forecast. Via statistical analysis of the ship movement state, the researchers utilize AR time series model to predict the pitch motion [2]. Another method is based on Kalman filtering algorithm, including system modeling, shaping filter design, system extension and other simulation studies. Time series analysis method is simple and quick, while the Kalman estimation method aiming at specific system gained the better prediction effects. Though the conditions of use are more, the forecast accuracy is high, and with wide application prospects [3]. There are many subsistent methods, but most of them are difficult to satisfy accurate and convenient conditions. Many scholars have adopted the idea of systematic identification [4] in recent years, and combined with the pitch experiment to analyze its law. Compared with other means, system identification combined with free-running model test or full-scale trials to 
determine the hydrodynamic derivatives in the mathematical models of ship steerable motion is easier and more effective [5]. With the development of identification technology and experimental measurement, this method has a broad application prospect.

In this paper, the author established system identifiable mathematical model and means based on genetic algorithms has been established, developed recognition software and verified the reliability of system identification [6]. Meanwhile, the author obtained the changing regularities of hydrodynamic coefficients with draft and initial tilt angle of the model in different drafts and initial tilt angles and their kinematic equation in the condition of still water. And the comparative analysis of the ship's pitch motion model and its hydrodynamic coefficient has been completed.

\section{Experimental Model and Scheme}

a) Experimental model

In the experiment, the model of the SWATH-USV was selected and carried out without sail, and the major parameters were showed below:

Table 1. Main dimensions of the model.

\begin{tabular}{lllll}
\hline $\begin{array}{l}\text { Sequence } \\
\text { number }\end{array}$ & Variate names & Symbol & Units & $\begin{array}{l}\text { Date } \\
\text { size }\end{array}$ \\
\hline 1 & Length over all & $\mathrm{L}$ & $\mathrm{m}$ & 1.2 \\
2 & Moulded breadth & $\mathrm{B}$ & $\mathrm{m}$ & 0.6 \\
3 & Moulded depth & $\mathrm{D}$ & $\mathrm{m}$ & 0.34 \\
4 & Moulded draft & $\mathrm{T}_{1}$ & $\mathrm{~m}$ & 0.14 \\
5 & Designed speed & $\mathrm{Vs}$ & $\mathrm{kn}$ & 5 \\
6 & Designed displacement & $\Delta$ & $\mathrm{kg}$ & 35 \\
\hline
\end{tabular}

b) Experimental equipment

The SWATH-USV model, MTi-G Inertial measurement instrument (which could measure the model's six degrees of freedom motion), data transmission line, SBC, weights.

c) Experimental steps

Because of the conditions, it is impossible to simulate the pitching motion of the model in waves in the experiment. So it simulates the motions of still water to determine its pitch motion.

1. After connecting the equipment, placed the craft mold in the center of the pool. After the craft stabilized, pressurize the load and adjust the ballast position to make it float. And then read its initial angle in still water.

2. In the case of design draft, manually apply force to the craft mold to give it an initial pitch angle. When the external force was released, MTi-G software was used to collect data until the ship model was stabilized and the data was collected and the pitch angle data was read.

3. Change the initial pitch angle and repeat step (2). The initial angle was given as 3, 5, 7 and 9 .

4. Change the draft of ship model by increasing or reducing the pressure load to 0.9 and 1.1 times respectively, and repeat the steps (2) and (3) in the water state of the two pools respectively.

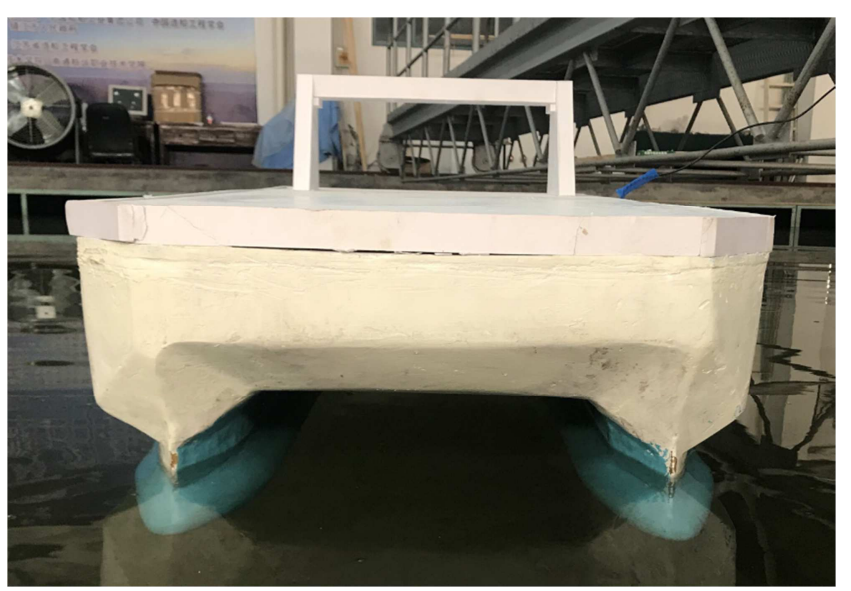

Figure 1. SWATH-USV model pitch test.

\section{Mathematical Model of Pitch Motion Identification of the SWATH-USV Model}

System identification is to determine a system equivalent to the identified system in a specified class of systems based on the observation of input and output. From a practical point of view, system identification is to select a model from a set of models, which can best fit the dynamic characteristics of the actual process in accordance with some rules. Whatever sense, system identification is based on the equivalence criteria of data model to achieve the purpose of mastering the laws of things, understanding things and using the results of identification to solve practical problems.

1. Righting moment

When the ship is pitching at an angle $\varphi$, the center of buoyancy and gravity are no longer perpendicular to each other, creating a moment to return the ship to its original position, which is called Righting moment $M(\varphi)$. Initial stability formula:

$$
M(\varphi)=-D h \varphi
$$

In the formula:

$\mathrm{D}$ is the displacement of ship;

$\mathrm{h}$ is the initial stability height of the ship.

2. Damping torque

Pitch damping is a function of angular velocity, which is generally expressed as:

$$
M(\dot{\varphi})=-2 N \dot{\varphi}-W|\dot{\varphi}| \dot{\varphi}-x \dot{\varphi}^{3}
$$

In the formula: $N 、 W$ and $x$ are pitch damping torque coefficient.

\section{Inertia moment}

The angular acceleration of the ship in the course of rotation is present, and the moment of inertia is inevitable. The longitudinal moment of inertia is composed of two parts, namely, the moment of inertia of hull itself and the moment of additional inertia. Generally, they are all linear with angular acceleration: 


$$
M(\ddot{\varphi})=-\left(I_{y y}+J_{y y}\right) \ddot{\varphi}=-I_{y y} \ddot{\varphi}
$$

Based on the principle of dynamic balance of objects, the balance condition of ship model is $\sum M=0$, so the equilibrium equation of the ship model in still water is:

$$
M(\ddot{\varphi})+M(\dot{\varphi})+M(\varphi)=0
$$

In this paper, the author transformed the restoring torque terms in the pitching equation in the form of one power, quadratic, cube and sinusoid respectively. After that finding the pitching equation which is suitable for the boat model in this paper.

Identification equation 1:

$\ddot{\varphi}+\frac{2 N \dot{\varphi}}{I^{\prime} y y}+\frac{W|\dot{\varphi}| \dot{\varphi}}{I^{\prime} y y}+\frac{x \varphi^{3}}{I^{\prime} y y}+\frac{C_{1}}{I^{\prime}{ }_{y y} \varphi^{3}}+\frac{C_{2}}{I^{\prime}{ }_{y y} \varphi^{2}}+\frac{C_{3}}{I^{\prime}{ }_{y y} \varphi}=0$

Identification equation 2 :

$$
\ddot{\varphi}+\frac{2 N \dot{\varphi}}{I^{\prime} y y}+\frac{W|\dot{\varphi}| \dot{\varphi}}{I^{\prime} y y}+\frac{x \varphi^{3}}{I^{\prime} y y}+\frac{D h \varphi}{I^{\prime} y y}=0
$$

Identification equation 3:

$\ddot{\varphi}+\frac{2 N \dot{\varphi}}{I^{\prime} y y}+\frac{W|\dot{\varphi}| \dot{\varphi}}{I^{\prime} y y}+\frac{x \varphi^{3}}{I^{\prime} y y}+\frac{C_{1}}{I^{\prime} y y(\sin \varphi)^{2}}+\frac{C_{2}}{I^{\prime} y y \sin \varphi}=0$

The design variables selected from the above equation are:

$$
I^{\prime}{ }_{y y}, \mathrm{~N}, \mathrm{~W}, \mathrm{x}, \mathrm{h}, \mathrm{C}_{1}, \mathrm{C}_{2}, \mathrm{C}_{3} .
$$

The range of values for design variables are:

$$
\begin{aligned}
& I^{\prime}{ }_{y y} \in[0,0.1], N \in[0,1], W \in[0,1], x \in[0,10], \\
& h \in[0,10], C_{1} \in[0,10], C_{2} \in[0,10], C_{3} \in[0,10]
\end{aligned}
$$

In the equation, $\varphi, \dot{\varphi}, \ddot{\varphi}$ are pitching angle, angular velocity in pitch, angular acceleration in pitch respectively; $I_{y y}$ is the total moment of inertia of hull pitching; $N$ is the pitch linear damping coefficient; $W$ is the pitch squared damping coefficient; $x$ is the pitch to the third power damping coefficient; $C_{1}, C_{2}$ are the nonlinear restoring torque coefficient; $C_{3}$ is the linear recovery moment coefficient.

Based on the above three identification models, the error estimation criteria at the moment $\mathrm{K}+1$ are as follows according to measuring the angle at the moment $\mathrm{K}$ :

$$
\varepsilon_{k+1}=\dot{\varphi}_{k+1}^{\prime}-\dot{\varphi}_{k+1}
$$

In the equation, $\dot{\varphi}_{K+1}$ is the measurement of the angular velocity at $\mathrm{K}+1$ moment; $\dot{\varphi}_{K+1}^{\prime}$ is the value of the identification result.

So formula (9) was selected as the target function. The smaller the value of the objective function, the better the formula fitting effect.

$$
F(x)=\sqrt{1 / N \sum_{k=1}^{N}\left(\dot{\varphi}_{K+1}^{\prime}-\dot{\varphi}_{K+1}\right)^{2}}
$$

\section{Pitch Identification Analysis}

\subsection{Comparative Analysis of Experimental Data}

In the longitudinal experiment of SWATH-USV, three experiments were carried out for each initial pitch angle under different draught conditions, and the best set of data was selected as valid data. The initial pitch angle in figure 2 is 3 , and the draft is 0.9 times the design draft, the design draft and 1.1 times the design draft respectively. Regardless of the error caused by human measurement factors, the composite law of pitch angular velocity attenuation is shown in the figure, so the pitch test data can be used.

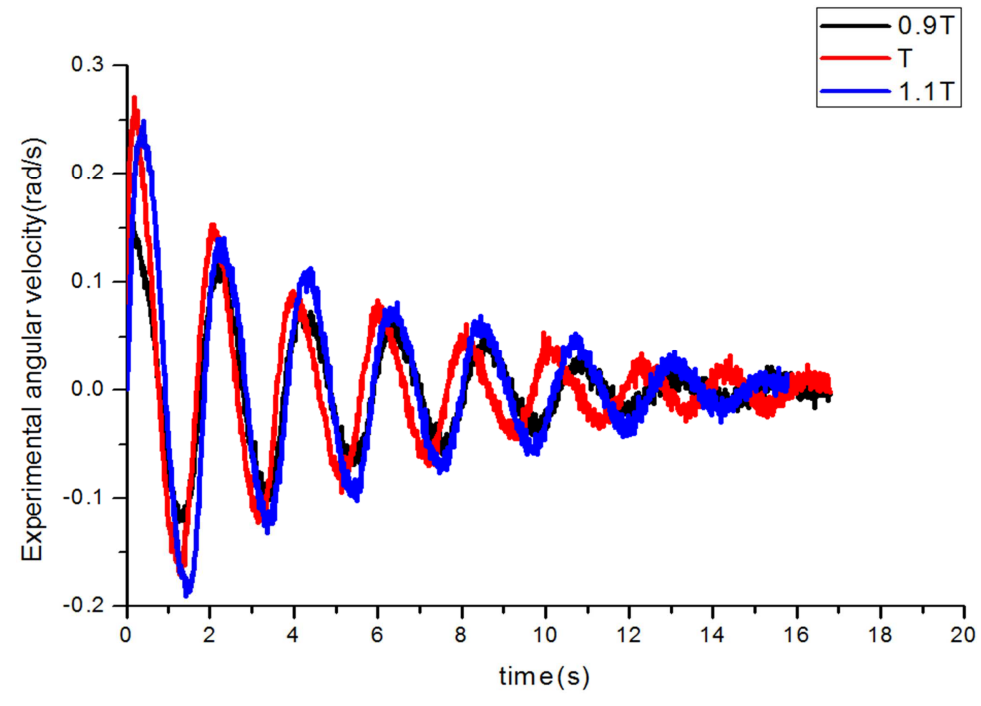

Figure 2. The change curve of angular velocity at initial pitch angle 3 of different draft.

As the figure 2 showed, when the initial pitch angle was 3 , the peak value of the pitch angle velocity increased with the increase of draft, and the pitch period increases, which indicated that the larger the draft increases, the worse its wave resistance was. In addition, it was found that the attenuation period of pitch of this model was longer than that of general monomer ships. On the one hand, it was due to the ship shape factor. During the pitching process, the pitch motion was 
accompanied by the roll motion, and the pitch cycle was relatively close to the roll cycle, forming the torsional rock. On the other hand, because of the arrangement factor, the placement of ballast made the center of gravity of the ship model high, which remained to be improved.

\subsection{Experimental Results and Analysis}

The author selected genetic algorithm and set the following basic parameters.

Population size was 400; Crossover probability was 0.7 ; Mutation probability was 0.15 ; The genetic factors were 0.05 , and the weight of evolution was 0.9 .

Three different initial pitch angles of different draft are identified and calculated by three identification systems with different pitch mathematical models. The identification results are shown in table 2 :

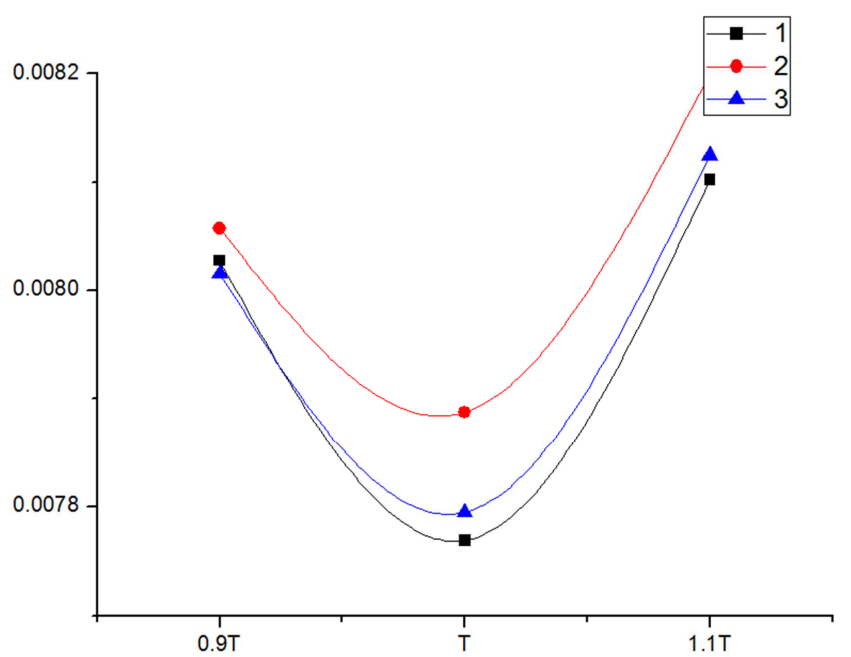

Figure 3. The value of the objective function was calculated under different draft conditions when the initial pitch angle is 3 .

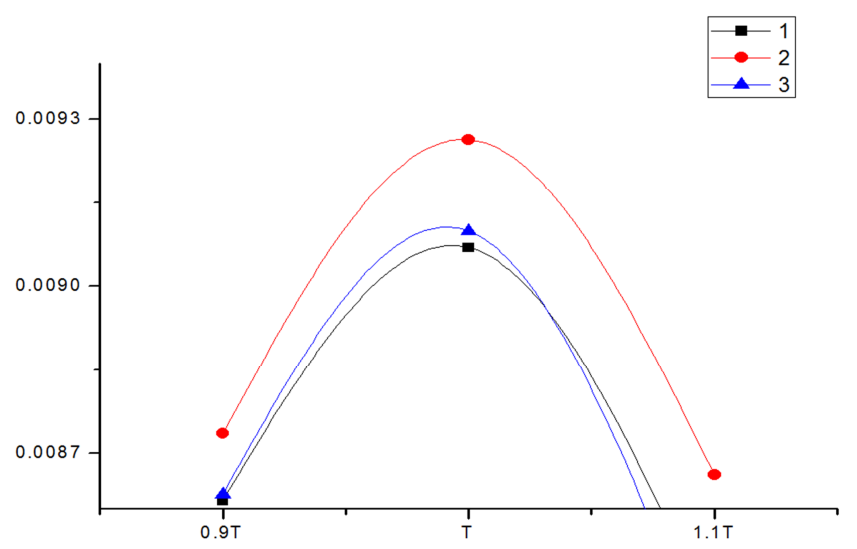

Figure 4. The value of the objective function was calculated under different draft conditions when the initial pitch angle is 5 .

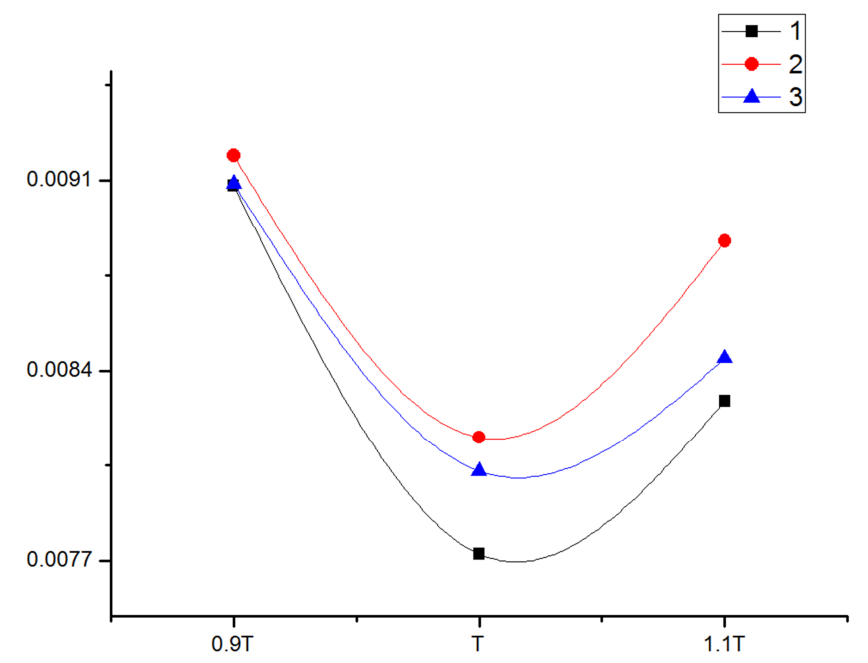

Figure 5. The value of the objective function was calculated under different draft conditions when the initial pitch angle is 7.

Table 2. The objective function values of the identification mathematical model.

\begin{tabular}{lllll}
\hline objective function values & & Function 1 & Function 2 & Function 3 \\
\hline \multirow{2}{*}{ initial pitch angles $3^{\circ}$} & $0.9 \mathrm{~T}$ & 0.008027143 & 0.008057216 & 0.008015505 \\
& $\mathrm{~T}$ & 0.007768651 & 0.007887628 & 0.007794842 \\
& $1.1 \mathrm{~T}$ & 0.008102280 & 0.008199922 & 0.008124685 \\
initial pitch angles $5^{\circ}$ & $0.9 \mathrm{~T}$ & 0.008615596 & 0.008735177 & 0.008626915 \\
& $\mathrm{~T}$ & 0.009069221 & 0.009264003 & 0.009097961 \\
& $1.1 \mathrm{~T}$ & 0.008394674 & 0.008661976 & 0.009291173 \\
initial pitch angles $7^{\circ}$ & $0.9 \mathrm{~T}$ & 0.009081213 & 0.009191506 & 0.008030085 \\
& $\mathrm{~T}$ & 0.007725706 & 0.008154394 & 0.008445253 \\
initial pitch angles & $1.1 \mathrm{~T}$ & 0.008288788 & 0.008876588 & 0.008626539 \\
$9^{\circ}$ & $0.9 \mathrm{~T}$ & 0.008633223 & 0.008732383 & 0.011912209 \\
\hline
\end{tabular}

By comparing the above objective function values, it was found that the objective function value of equation 1 was the minimum under the conditions of three different draught and four different initial pitch angles, indicating that function 1 had the best fitting effect.

Table 3. The optimum values of design variables identified by equation 1 .

\begin{tabular}{llll}
\hline variable parameters & lower limit & upper limit \\
\hline total moment of inertia of hull pitching $I_{v v}$ & 0 & 0.1 & optimal value \\
pitch linear damping coefficient $N$ & 0 & 1 & 0.0937801 \\
pitch squared damping coefficient $W$ & 0 & 1 & 0.0950886 \\
\hline
\end{tabular}




\begin{tabular}{llll}
\hline variable parameters & lower limit & upper limit \\
\hline pitch to the third power damping coefficient $x$ & 0 & 10 & optimal value \\
nonlinear restoring torque coefficient $C_{1}$ & 0 & 10 & 0.0046185 \\
nonlinear restoring torque coefficient $C_{2}$ & 0 & 10 & 0.8210960 \\
linear recovery moment coefficient $C_{3}$ & 0 & 10 & 0.0015652 \\
\hline
\end{tabular}

Taking pitch angle 5 under design draft as an example, the obtained identification equation is:

$$
\dot{\varphi}+\frac{0.19018 \dot{\varphi}}{I^{\prime}{ }_{y y}}+\frac{0.01326|\dot{\varphi}| \dot{\varphi}}{I^{\prime}{ }_{y y}}+\frac{0.00462 \varphi^{3}}{I^{\prime}{ }_{y y}}+\frac{0.82110}{I^{\prime}{ }_{y y} \varphi^{3}}+\frac{0.00157}{I^{\prime}{ }_{y y} \varphi^{2}}+\frac{0.69775}{I^{\prime}{ }_{y y} \varphi}=0
$$

Table 4. Error table of experimental and identification results.

\begin{tabular}{llll}
\hline Time (s) & Experimental angular velocity & Fitting angular velocity & Error \\
\hline 0.81 & -0.297958089 & -0.304524846 & $2.2 \%$ \\
0.82 & -0.2980017 & -0.297995277 & $0.002 \%$ \\
0.83 & -0.305448733 & -0.297829576 & $2.49 \%$ \\
0.84 & -0.303303067 & -0.305003833 & $0.56 \%$ \\
0.85 & -0.309937189 & -0.302662427 & $2.35 \%$ \\
0.86 & -0.305324878 & -0.309026728 & $1.21 \%$ \\
0.87 & -0.304989944 & -0.304236879 & $0.25 \%$ \\
0.88 & -0.303067567 & -0.303690749 & $0.21 \%$ \\
0.89 & -0.301933678 & -0.301571049 & $0.12 \%$ \\
0.9 & -0.298614 & -0.300234398 & $0.54 \%$ \\
\hline
\end{tabular}

In order to analyze the error between the experimental value and the fitting value, the experimental value of the angular velocity in the state of initial pitch angle 7 of the design draft was compared with the fitting value identified by equation 1 . The result is showed as figure 6:

According to figure 6, it can be seen that the fitting effect of experimental angular velocity and identified angular velocity is better. As can be seen from table 4, the error of experimental angle and identified angular velocity is less than $3 \%$, indicating that the software is reliable and can predict the angular velocity at the next moment.

Through the identification calculation of 12 sets of longitudinal experimental data, the total moment of inertia of hull pitch $I_{y y}$ and the linear damping moment coefficient $N$ square damping $W$, third damping $x$ and each restoring moment coefficient are obtained in each case.

In the condition of three different draught, the variation curves of the variables with the initial pitch Angle are shown in the following figure:

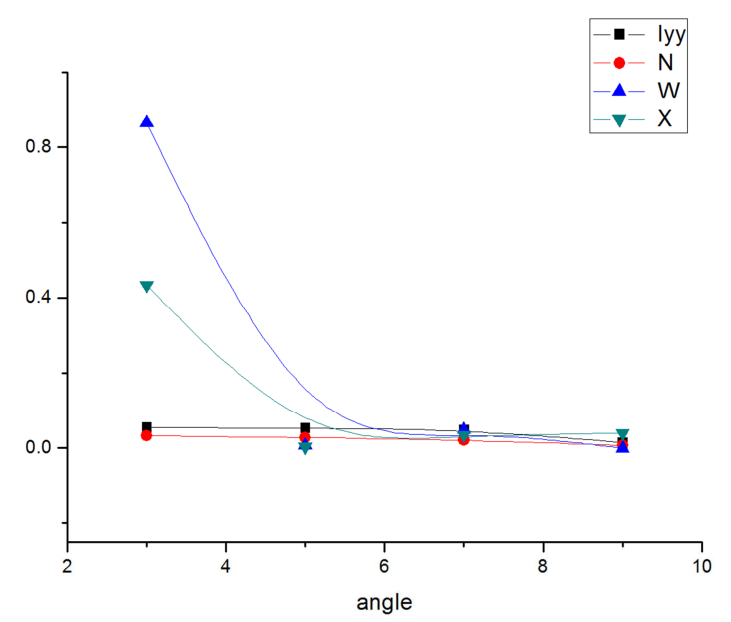

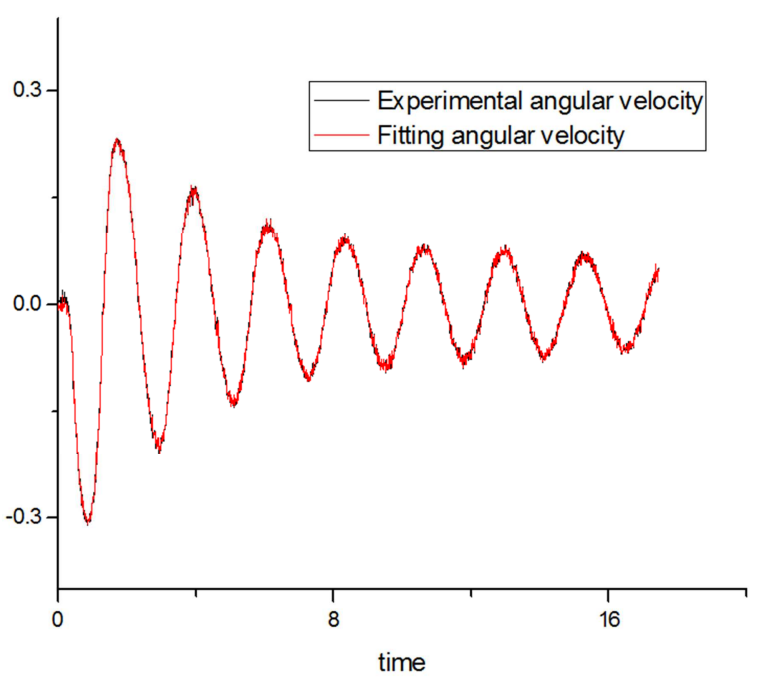

Figure 6. Experimental angular velocity and fitting angular velocity curves.

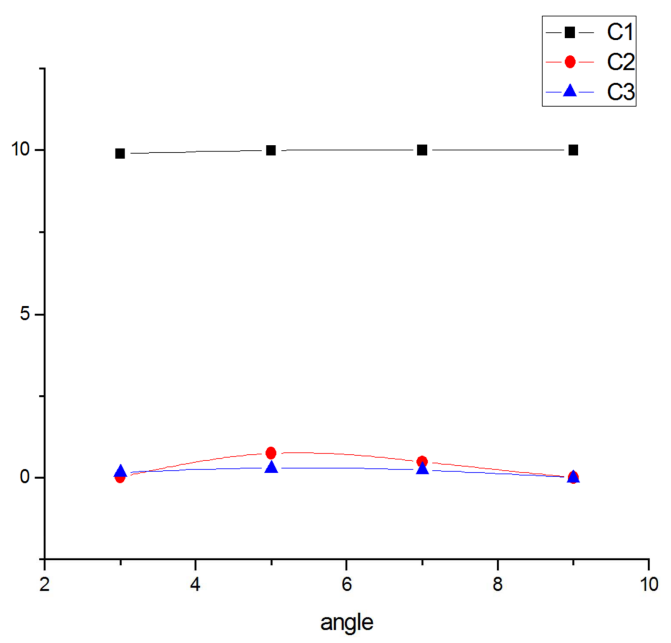

Figure 7. The curves of moment coefficients change with the initial pitch angle under 0.9 times the design draft. 

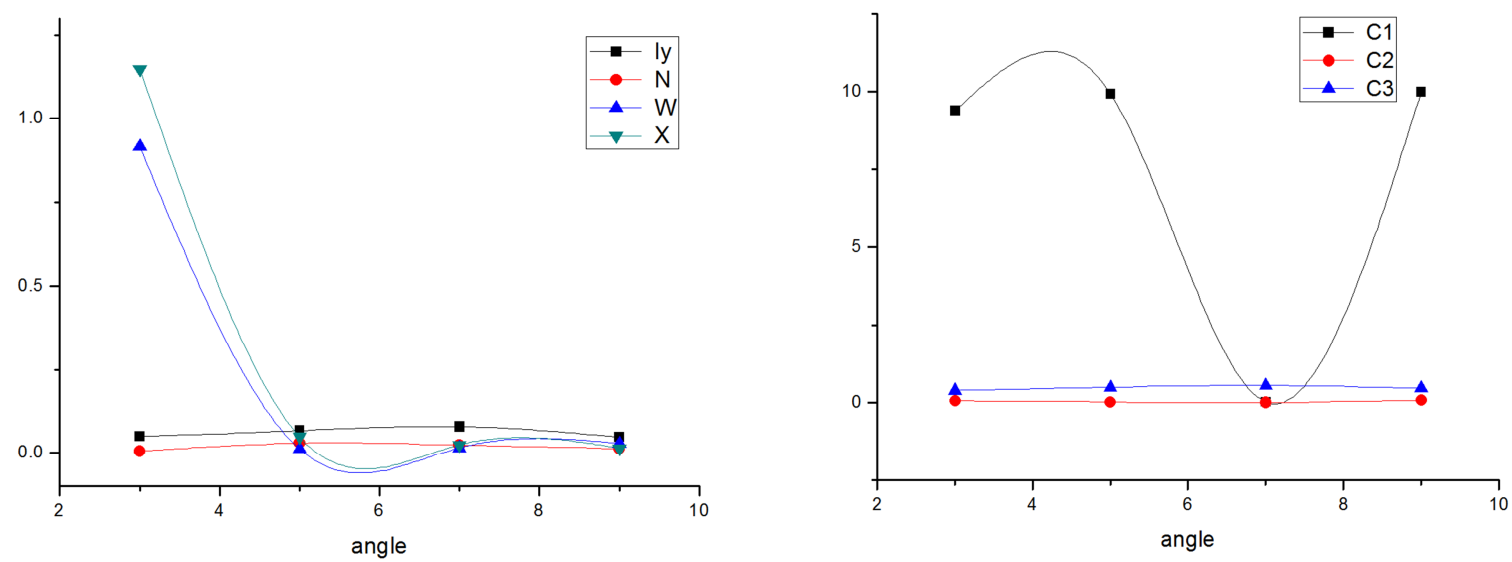

Figure 8. The curves of moment coefficients change with the initial pitch angle under the design draft.
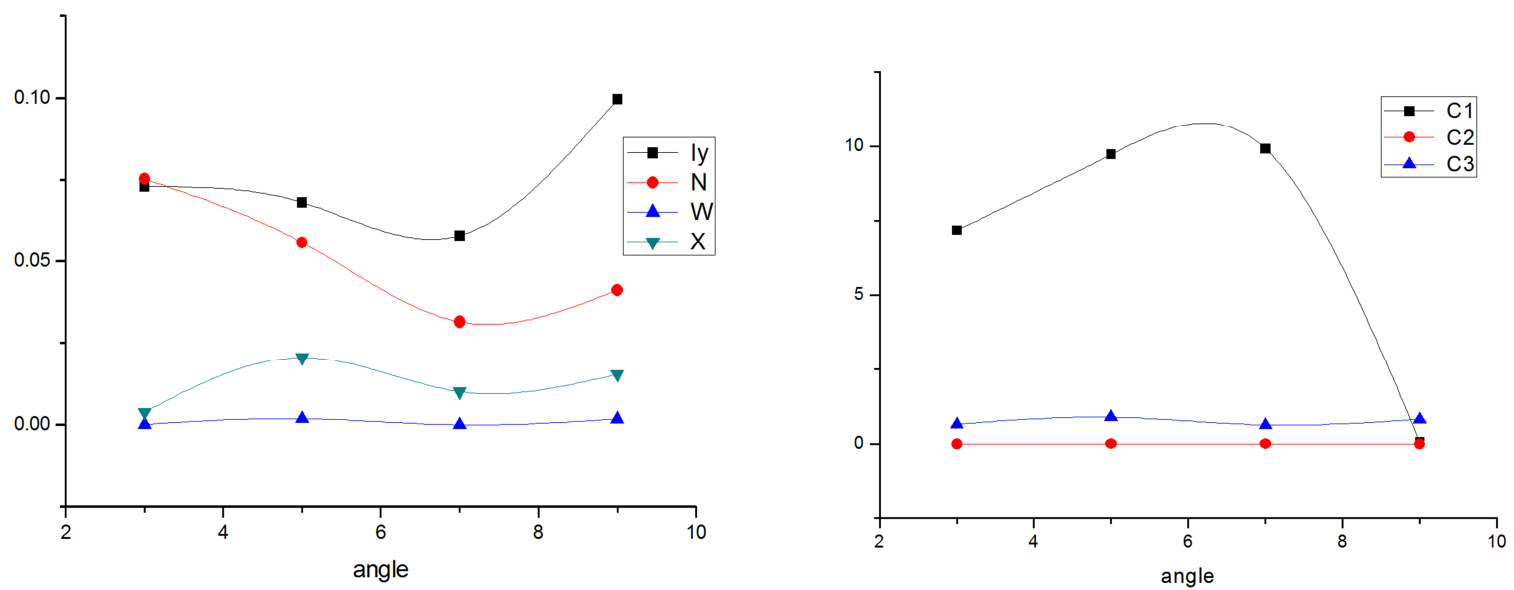

Figure 9. The curves of moment coefficients change with the initial pitch angle under 1.1 times the design draft.

It can be seen from figure 7-9 that the value of each moment coefficient will change with the change of draft and initial pitch angle.

Under the condition of 0.9 times the design draft, hull pitching the total moment of inertia $I_{y y}$ and pitch damping moment coefficient linear $N$ only slightly with the increase of initial pitch angle smaller trend, and the overall basic flat. The square damping coefficient $W$ and the damping coefficient of the third power $x$ is drastically with the increase of initial pitch angle decreases, and the final stable close to zero; Relative nonlinear restoring moment coefficient $C_{l}$ remains in a large and stable state. Relative non-linear restoring moment coefficient $C_{2}$ has a slight change trend of increasing first and then decreasing, while relative linear restoring moment coefficient $C_{3}$ changes tend to be stable.

Under the condition of the design draft, hull pitching moment of inertia $I_{y y}$, pitch damping moment coefficient $N$, square linear damping coefficient $W$ and the tendency of the third power damping coefficient $x$ basic agree with 0.9 times the design draft, but square damping coefficient $W$ and the damping coefficient of the third power of $x$, with the increase of initial pitch Angle decreases the size of the more wins, and ultimately have a tiny increasing trend; Relative nonlinear restoring moment coefficient $C_{l}$ fluctuates greatly, first increases, then decreases, and finally increases. The variation trend of relative nonlinear restoring moment coefficient $C_{2}$ and relative linear restoring moment coefficient $C_{3}$ is basically consistent with that of the design draft of 0.9 .

Under the condition of 1.1 times the draught, hull pitching moment of inertia $I_{y y}$, pitching damping coefficient of the linear damping moment coefficient $N$, power three $x$ are not stable, has the certain fluctuation, in which hull longitudinal total moment of inertia and longitudinal wave linear damping moment coefficient $N$ decrease then increase first and third power damping coefficient $x$ increases first and then decrease then increase, and square damping coefficient $W$ is almost close to zero and maintained steady; Compared with $C_{l}$, the value of the nonlinear restoring torque coefficient is always larger, with the change trend of first increasing and then decreasing. The change of $C_{3}$ is always stable and close to 0 .

The results show that the nonlinear damping proportion is very low in the pitch motion with small angle, and the relative nonlinear restoring torque coefficient $C_{l}$ has a great influence on the SWATH-USV pitching.

\section{Conclusion}

In this paper, model experiment and system identification method were used to study the pitching-attenuation motion mode of a SWATH-USV in still water state. The mathematical 
model which was most suitable for the ship model in the paper and could accurately describe the pitch motion process in 12 working conditions was found through the calculation of identification program. The correct value of each moment coefficient was obtained, the law of each moment coefficient changing with the angle of draft and pitch was found, and the correctness of the system identification software was verified. It can be concluded that the system identification software can predict the future navigation parameters and motion posture of unmanned boat, providing reference for further study on the pitching motion of SWATH-USV. The prediction method in this paper is also applicable to the prediction of rolling motion of ships.

\section{Acknowledgements}

This subject is funded by the following project:

National Natural Science Foundation of China: Comprehensive optimization model and method for the performance of high speed USV, project approval number: 51379094.

\section{References}

[1] Fan Wu, Zhijian Chen, Jubin Liu, Yansong Lv. Hydrodynamic calculation of SWATH in waves [J]. Ship Engineering, 2004.

[2] Jie Ma. A comparative study of AR method and multilayer hierarchical method for ship pitch motion prediction $[\mathrm{M}]$. Proceedings of China intelligent automation conference in 2009 (volume 2), 2009.

[3] Lee C M, CURPHEY R M. Prediction of motion, stability, and wave load of small-waterplane-area twin-hull ships [J]. Trans,
SNAME, 1977, (85): 94- 130.

[4] Feng Zhu, Songlin Yang. A preliminary study on the system identification methods of pitch and heave motion of a composite trimara [J], 2014.

[5] Heng Zhang, Jide Li, Xiaodong Zhao. The nonlinear motion response of ship is solved based on system identification method [J]. Journal of Dalian Maritime University, 2008.

[6] Baohua Wang. Research on the prediction and control strategy of SWATH [D]. Harbin Engineering University, 2013.

[7] Zhu Hong. Study on the nonlinear dynamic characteristics of ship under the crosswise rolling coupling [D]. Jiangsu University of Science and Technology, 2016.

[8] D Dumitru, Numerical investigation of a two - degrees - of freedom ship model for pitch - roll motion [C], IOP Conference Series: 10.1088/1757-899X/145/8/082007,2016.

[9] Jun Zhu, Kunlun Huang, Liushuai Cao. Response model of velocity oscillations to ship pitch and heave coupled motions [J]. Journal of Naval University of Engineering, 2012.

[10] Wenqiang Shi, Xianzhao Yu. Development status and trend of SWATH abroad [J]. Ship Science and Technology, 2012.

[11] Bowen Shi. Prediction of ship wave resistance based on real wave spectrum and CFD [D]. Dalian Maritime University, 2016.

[12] Kui Ye. Research on anti-pitch control method of ship rudder [D]. Harbin Engineering University, 2004.

[13] Ping Cheng, Yong Ai, Decheng Wan. Unsteady hydrodynamic simulation of a floating offshore wind turbine with oscillating pitch motion [M]. Proceedings of the second global Chinese hydrodynamics academic conference (next volume), 2016. 